Proc. Indian Acad. Sci. (Earth Planet. Sci.), Vol. 93, No. 1, March 1984, pp. 37-45.

(c) Printed in India.

\title{
On the exact evaluation of displacements within a semi-infinite solid due to a buried line source
}

\author{
SATYESH K BHANDARI* and RAMESH CHANDER \\ Department of Earth Sciences, University of Roorkee, Roorkee 247667 , India \\ * Present address: Oil and Natural Gas Commission, Jodhpur 342001, India \\ MS received 4 March 1983; revised 8 December 1983
}

\begin{abstract}
Garvin's variant of the Cagniard technique has been used to evaluate exactly the displacements at points within a homogeneous, isotropic, perfectly elastic, solid half space due to a buried explosive line source. The disturbance at any given time is confined to a region bounded by the free surface of the half space and the $P$ wave front within it. The disturbance associated with the Rayleigh pulse affects a smaller region bounded by the free surface and the PP wave front.
\end{abstract}

Keywords. Lamb problem; exact solution; Cagniard technique.

\section{Introduction}

Elastic wave propagation problems have been solved exactly in only a few cases. These stem mainly from the work of Cagniard (1962) and pertain to transient line sources in either an elastic solid half space or to two such half spaces in contact. The solutions for transient point sources in such media are available in the form of convolutional integrals. Garvin (1956) used a variant of the Cagniard technique to obtain exact wave forms expected at points of observation on the surface of an elastic solid half space due to a transient explosive source buried at some depth in it. We display in this paper the exact nature of wave motion within the half space in the same case.

\section{The problem}

The problem may be stated as follows. Consider a cartesian rectangular coordinate system $0 x y z$ (figure 1). A homogeneous, isotropic, perfectly elastic, solid half space is bounded by the $x y$ plane and occupies the region of the positive $z$ axis. An explosive line source is oriented parallel to the $y$ axis and intersects the $z$ axis at $z=h$. The source behaves as a unit step function in time. It is required to find the ensuing disturbance of the medium assuming that its surface is stress free and the radiation condition holds.

\section{The solution}

Let $V_{P}$ and $V_{S}$ be the compressional and shear wave speeds in the medium. Let $p$ and $k$ be the Laplace and spatial Fourier transform variables. Let $S_{x}(k, z, p)$ and $S_{z}(k, z, p)$ be 


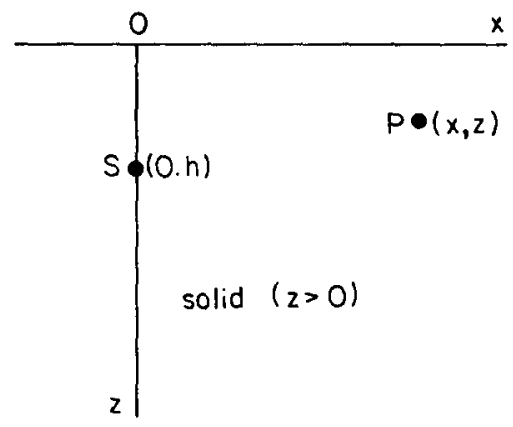

Figure 1. The coordinate system and the domain of the solid medium. $y$ axis points out of the paper but the $y$ coordinate is suppressed because of the two dimensional nature of the problem. $S$ represents the line source and $P$ the point of observation.

the Laplace (with respect to time) and Fourier (with respect to $x$ ) transformed $x$ and $z$ components of displacement $X(x, z, t)$ and $Z(x, z, t)$ respectively. Then (see Garvin 1956; and Bhandari 1982),

$$
\begin{aligned}
S_{x}(k, z, p)= & S_{x P} p^{-1} \exp \left[-(h-z) n_{P}\right]+S_{x P P} p^{-1} \exp \left[-(h+z) n_{P}\right] \\
& +S_{x P S} p^{-1} \exp \left(-h n_{P}-z n_{S}\right) \\
S_{z}(k, z, p)= & S_{z P} p^{-1} \exp \left[-(h-z) n_{P}\right]+S_{z P P} p^{-1} \exp \left[-(h+z) n_{P}\right] \\
& +S_{z P S} p^{-1} \exp \left(-h n_{P}-z n_{S}\right) .
\end{aligned}
$$

Here, $\quad S_{x P}(k, z, p)=j k / 4 n_{P} ; S_{x P P}(k, z, p)=j k B / 4$;

$$
\begin{aligned}
& S_{x P S}(k, z, p)=-j C n_{S} / 4 ; S_{z P}(k, z, p)=-1 / 4 ; \\
& S_{z P P}(k, z, p)=n_{P} B / 4 ; S_{z P S}(k, z, p)=-k C / 4 ; \\
& B=-1 / n_{P}-8 k^{2} n_{S} / D ; \\
& C=-4 k\left(2 k^{2}+p^{2} / V_{S}^{2}\right) / D ; \\
& D=\left(2 k^{2}+p^{2} / V_{S}^{2}\right)^{2}-4 k^{2} n_{P} n_{S} ; n_{P}=\left(k^{2}+p^{2} / V_{P}^{2}\right)^{1 / 2} ; \\
& n_{S}=\left(k^{2}+p^{2} / V_{S}^{2}\right)^{1 / 2} ; \text { and } j=(-1)^{1 / 2} .
\end{aligned}
$$

The significance of the subscripts $P, P P$, and $P S$ on $S_{x}$ and $S_{z}$ is that the respective terms are subsequently seen to yield contributions to the total displacement field as follows. $S_{x P}$ and $S_{z P}$ represent contributions to the displacement field due to compressional waves travelling from the source direct to the observation points. $S_{x P P}$ and $S_{z P P}$ yield contributions arising from the interaction of compressional waves from the source with free surface of the medium and the compressional waves resulting therefrom. $S_{x P S}$ and $S_{z P S}$ similarly represent the shear waves resulting from the interaction of compressional waves from the source with the medium boundary.

At this stage, Garvin set $z=0$ since his interest was in observations at the surface of the medium. As a result, there was only one exponential factor in each of equations (1) and (2). He then obtained the displacement components $X(x, 0, t)$ and $Z(x, 0, t)$ using the transformation from $k$ to $t$ suggested by Cagniard (1962). Since $z$ is non-zero for points within the medium, (1) and (2) contain three distinct exponential factors in the 
three terms. We thus had to apply Garvin's procedure thrice. We made the logical assumption that the time $t$ in the three transformations was the same. We reproduce here the results.

$$
\begin{aligned}
X(x, z, t) & =0 & & t \leqslant r / V_{P} \\
& =X_{P}+X_{P P}+X_{P S} & & t>r / V_{P} \\
Z(x, z, t) & =0 & & t \leqslant r / V_{P} \\
& =Z_{P}+Z_{P P}+Z_{P S} & & t>r / V_{P}
\end{aligned}
$$

Here, $\quad X_{P}(x, z, t)=\operatorname{Re}\left[j K_{P} Q_{P} / 2 a_{P}\right]$;

$$
\begin{aligned}
& X_{P P}(x, z, t)=\operatorname{Re}\left[-j\left\{K_{P P} / a_{P P}+8 K_{P P}^{3} b_{P P} / D_{P P}\right\} Q_{P P} / 2\right] ; \\
& X_{P S}(x, z, t)=\operatorname{Re}\left[j 2 K_{P S}\left(2 K_{P S}^{2}+V_{S}^{-2}\right) Q_{P S} / D_{P S}\right]
\end{aligned}
$$$$
Z_{P}(x, z, t)=\operatorname{Re}\left[-Q_{P} / 2\right]
$$$$
Z_{P P}(x, z, t)=\operatorname{Re}\left[-\left\{1+8 K_{P P}^{2} a_{P P} b_{P P} / D_{P P}\right\} Q_{P P} / 2\right] \text {; }
$$$$
Z_{P S}(x, z, t)=\operatorname{Re}\left[2 K_{P S}^{2} Q_{P S}\left(2 K_{P S}^{2}+V_{S}^{-2}\right) / D_{P S}\right] \text {; }
$$$$
Q_{P}=\left[j x+(h-z) K_{P} / a_{P}\right]^{-1} \text {; }
$$$$
Q_{P P}=\left[j x+(h+z) K_{P P} / a_{P P}\right]^{-1} \text {; }
$$$$
Q_{P S}=\left[j x+K_{P S}\left(h / a_{P S}+z / b_{P S}\right)\right]^{-1} \text {; }
$$$$
a_{i}=\left(K_{i}^{2}+V_{P}^{-2}\right)^{1 / 2}, i \neq P, P P, P S ;
$$$$
b_{i}=\left(K_{i}^{2}+V_{S}^{-2}\right)^{1 / 2}, i=P, P P, P S \text {; }
$$$$
K_{P}=\left[-j x t+(h-z)\left\{t^{2}-\left[x^{2}+(h-z)^{2}\right] V_{P}^{-2}\right\}\right] /\left[x^{2}+(h-z)^{2}\right] ;
$$$$
K_{P P}=\left[-j x t+(h+z)\left\{t^{2}-\left[x^{2}+(h+z)^{2}\right] V_{P}^{-2}\right\}\right] /\left[x^{2}+(h+z)^{2}\right] \text {; }
$$

$K_{P S}$ is the solution of $j K_{P S} x+h a_{P S}+z b_{P S}=t$;

$$
D_{i}=\left(2 K_{i}^{2}+V_{S}^{-2}\right)^{2}-4 K_{i}^{2} a_{i} b_{i}, i=P P \text { and } P S ;
$$

and

$$
r=\left[x^{2}+(h-z)^{2}\right]^{1 / 2} \text {. }
$$

Re stands for the real part of the following complex quantity.

$X_{P}$ and $Z_{P}$ have non-zero real parts for $t$ greater than $r / V_{P} . X_{P P}$ and $Z_{P P}$ have nonzero real parts for $t$ greater than $\left[x^{2}+(h+z)^{2}\right]^{1 / 2} / V_{P}$. Finally, $X_{P S}$ and $Z_{P S}$ have nonzero real parts at times greater than that required for a wave travelling as compressional wave from source to free surface and as shear wave after reflection there to reach the observation point.

\section{Results}

There are two distinct graphical ways to portray wave motion in a medium. Firstly, one may draw graphs of $x$ and $z$ displacement components as functions of time at a fixed point in the medium. Secondly, one may prepare maps of displacement components or total displacement at different points in the medium at a fixed time. We have adopted 
the latter approach. The computations were carried out after making the quantities appearing in the formulae suitably non-dimensional. Figures 2 to 5 are maps of the displacement field for $t=40$ time units after the source initiated, assuming $V_{P}=1$ velocity unit. The location of the source is marked by a blank spot in the upper left hand corners of figures 2,3 , and 5 . The different symbols in these figures represent the magnitudes of total displacement or displacement components at the respective points in the medium according to the scheme of table 1.

Figure 2 is a map of $\left(X_{P}^{2}+Z_{P}^{2}\right)^{1 / 2}$. This displacement field is due to the compressional wave direct from the source. Since $V_{P}$ is 1 velocity unit, the direct compressional disturbance can reach in 40 time units only those parts of the medium that lie within $\mathbf{4 0}$ length units radially from the source. This is vividly brought out in the figure where the blank area in the lower right corner represents points outside this distance limit. Computation of displacement was attempted at such points of the map frame also but for reasons indicated in the preceding section no contribution was obtained. The

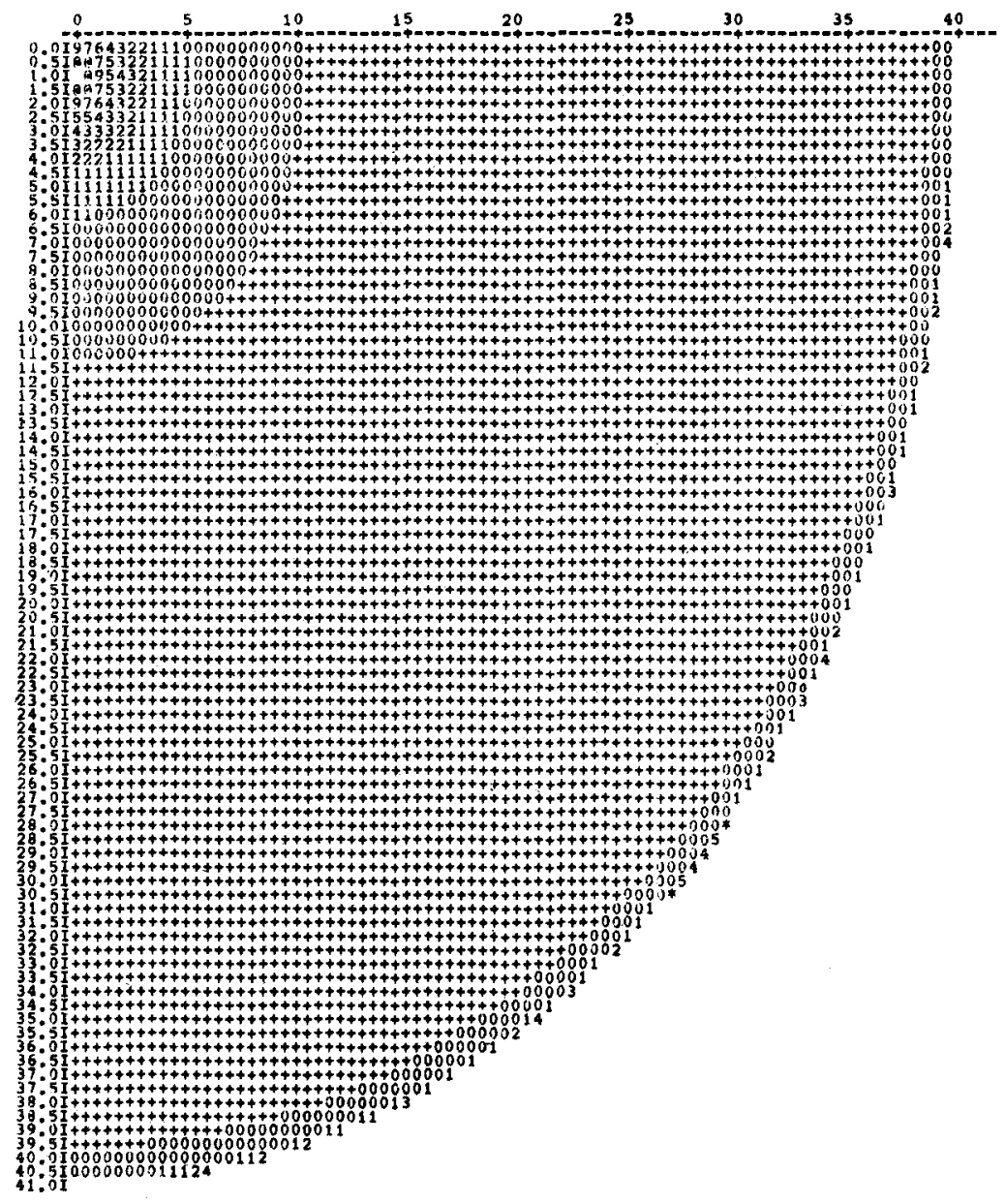

Figure 2. The total displacement $\left(X_{P}^{2}+Z_{P}^{2}\right)^{1 / 2}$ due to direct compressional wave from the source. The computations pertain to $t=40$ time units. The symbols are explained in table 1 . 
Table 1. List of symbols used in figures 2 to 5 .

\begin{tabular}{|c|c|c|c|}
\hline $\begin{array}{l}\text { Range of magnitude of total } \\
\text { displacement or displacement } \\
\text { component (normalized) }\end{array}$ & Symbol & $\begin{array}{l}\text { Range of magnitude of total } \\
\text { displacement or displacement } \\
\text { component (normalized) }\end{array}$ & Symbol \\
\hline 0.00 & (Blank) & $-0.00--0.05$ & A \\
\hline $0.00-0.05$ & + & $-0.05--0.10$ & B \\
\hline $0-05-0-10$ & $\mathbf{0}$ & $-0 \cdot 10--0 \cdot 15$ & C \\
\hline $0.10-0.15$ & 1 & $-0.15--0.20$ & D \\
\hline $0-15-0.20$ & 2 & $-0.20--0.25$ & $\mathrm{E}$ \\
\hline $0.20-0-25$ & 3 & $-0.25--0.30$ & $\mathbf{F}$ \\
\hline $0-25-0-30$ & 4 & $-0.30--0.35$ & $\mathbf{G}$ \\
\hline $0-30-0-35$ & 5 & $-0.35--0.40$ & $\mathbf{H}$ \\
\hline $0-35-0-40$ & 6 & $-0.40--0.45$ & I \\
\hline $0-40-0-45$ & 7 & $-0.45--0.50$ & $\mathbf{K}$ \\
\hline $0-45-0-50$ & 8 & $-0.50--0.55$ & $\mathbf{M}$ \\
\hline $0.50-0.55$ & 9 & $-0.55--0.60$ & $\mathbf{P}$ \\
\hline $0.55-0.60$ & * & less than -0.60 & $\mathbf{S}$ \\
\hline larger than 0.60 & @ & & \\
\hline
\end{tabular}

surface bounding the disturbed region will be called the $P$ wave front in the sequel. It is also observed from figure 2 that the total displacement is quite large in the vicinity of the source where a singularity is expected. The total displacement is also seen to be large at several irregularly spaced points near the $P$ wave front. Actually, a symmetry about the source is expected and would have been obtained if the points for calculating the displacements had been chosen to lie on a polar grid rather than the rectangular grid of the figure. Other calculations, not shown here, indicate that the $P$ wave front is a surface of discontinuity in the present case, and on its one side the medium is at rest while on its other side large displacements are encountered but they die down rapidly with distance from the wave front. Displacements are small but not zero between these two regions of high values.

Figure 3 is a map of $\left(X_{P P}^{2}+Z_{P P}^{2}\right)^{1 / 2}$. This contribution to the displacement field is due to the compressional wave type disturbance arising out of the interaction of the direct compressional wave from the source with the bounding surface of the medium. A comparison of figures 2 and 3 reveals that the region of disturbance is slightly smaller in the latter case than in the former. More precisely the disturbed region is bounded by the $P P$ wave front which is a cylindrical surface of radius 40 length units coaxial with the image line source parallel to the actual source but passing through the $z$ axis at $z=-1$ length unit. Further comparison of the figures reveals that the distribution of displacements in the disturbed regions is more complicated in figure 3 than in figure 2. Notably the comparatively large displacements at points near the surface with $x$ coordinates of about 20 length units is due to the Rayleigh type disturbance ascribable numerically to the smallness of $D_{P P}$ (Rayleigh function in the second terms of expressions for $X_{P P}$ and $Z_{P P}$ ). Influence of this function is felt throughout the region enclosed by the $P P$ wave front.

Figure 4 is a map of $\left(X_{P S}^{2}+Z_{P S}^{2}\right)^{1 / 2}$. This displacement field is due to the shear wave type disturbance arising out of the interaction of direct compressional wave from the source with the bounding surface of the medium. The extent of the disturbed region is 


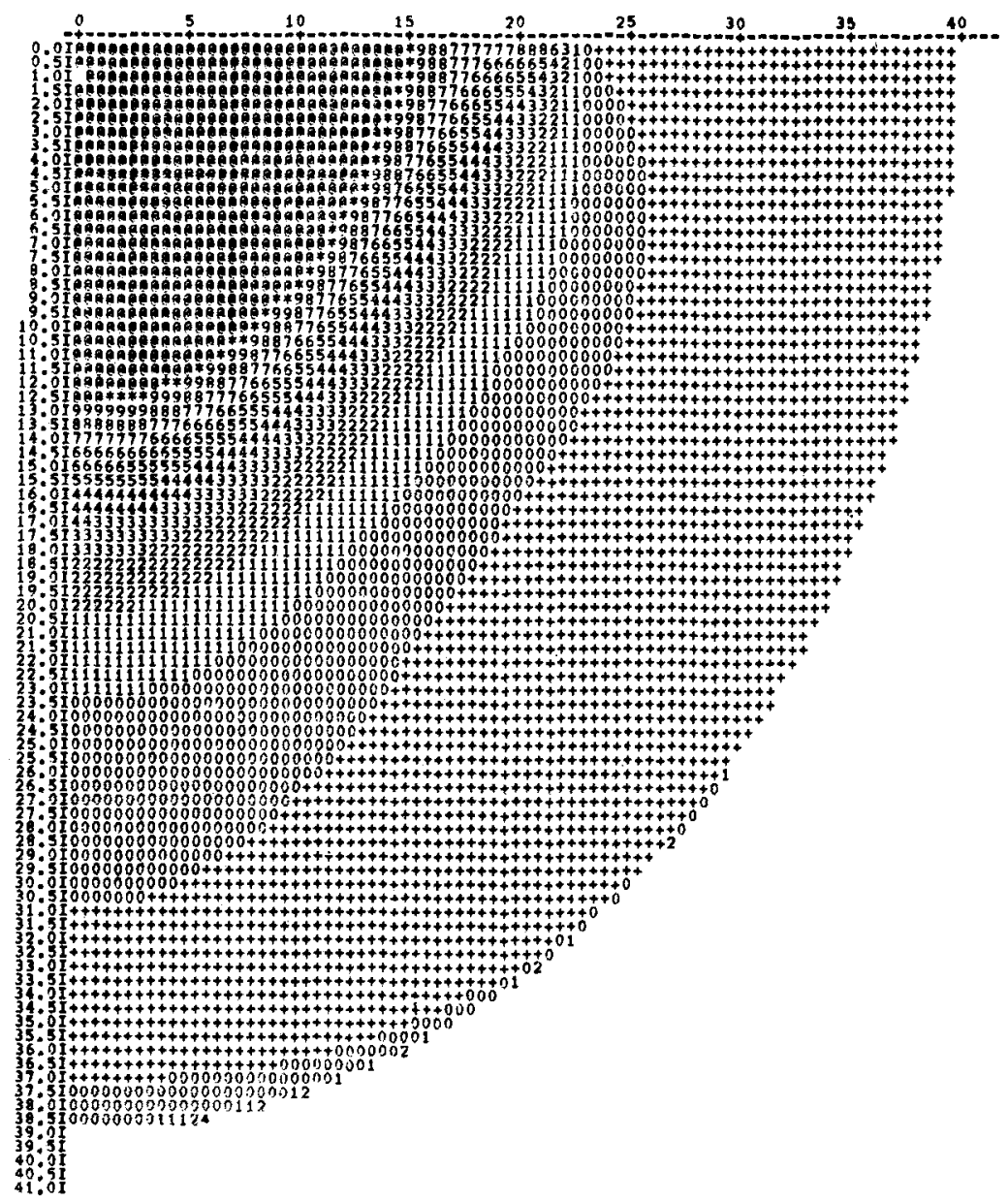

Figure 3. The total displacement $\left(X_{P P}^{2}+Z_{P P}^{2}\right)^{1 / 2}$ due to compressional wave reflected at the medium boundary.

even smaller than in figure 3. A notable feature of the disturbed region is that over a considerable part it is bounded by a plane parallel to the line source and making an angle of $\operatorname{arc} \tan \left(V_{S} / V_{P}\right)$ with the $x y$ plane. This is clearly a head wave front. The distribution of displacement in the disturbed region is again quite complex. The points of the medium lying near its bounding surface and having $x$ coordinates of about 20 length units again experience large displacements ascribable numerically to the smallness of $D_{P S}$. Finally, there is no disturbance along the $y z$ plane because there is no $P$ to $S$ mode conversion at the $y$ axis.

Figure 5 is a map of $\left(X^{2}+Z^{2}\right)^{1 / 2}$. The region of disturbance coincides with that of the direct compressional pulse from the source. The region of disturbance due to $P S$ type pulse is quite prominent. The region disturbed strongly by the Rayleigh pulse stands out quite clearly in figure 5. 


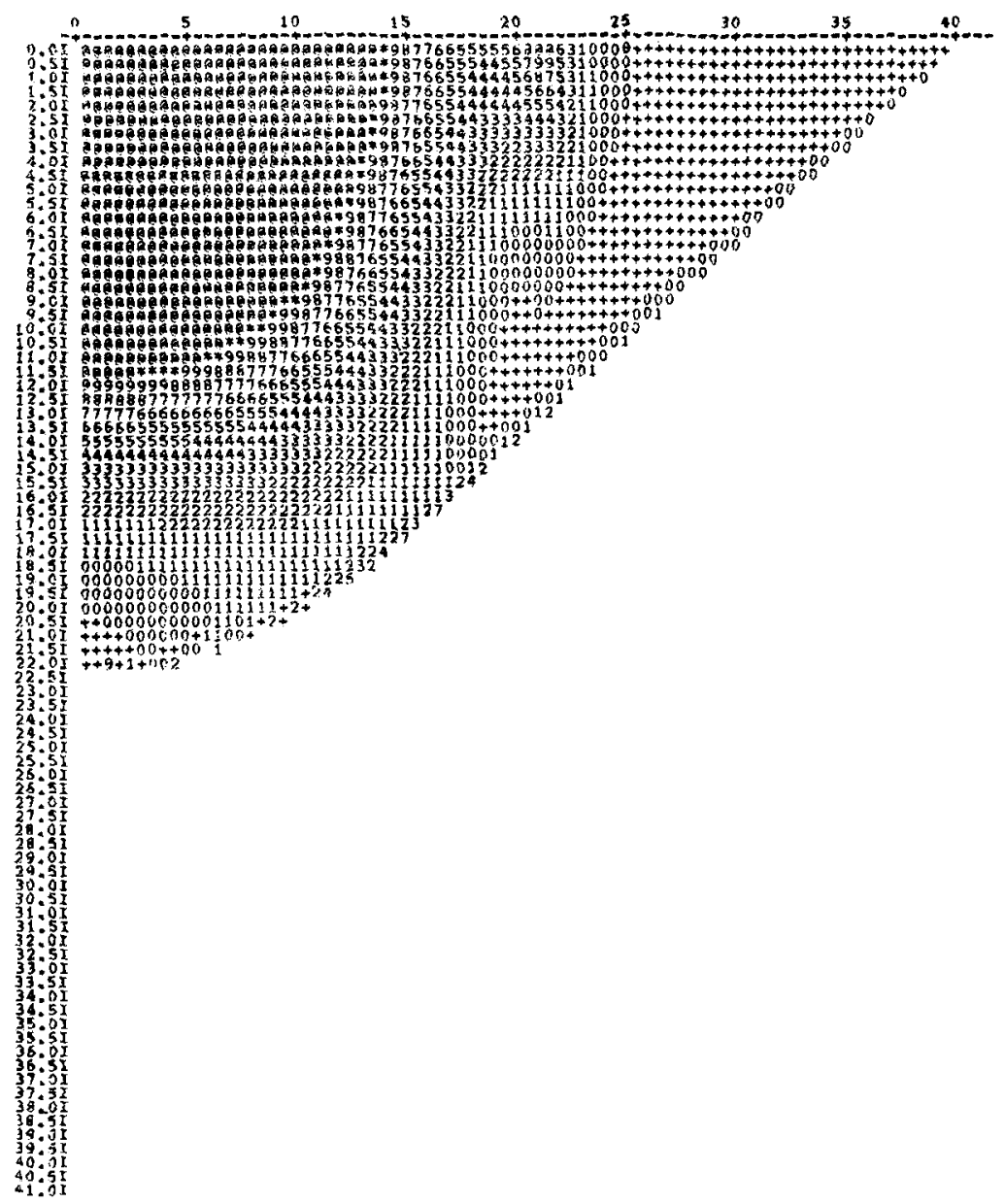

Figure 4. The total displacement $\left(X_{P S}^{2}+Z_{P S}^{2}\right)^{1 / 2}$ due to shear wave arising from compressional wave reflected at the medium boundary.

\section{Concluding discussion}

The disposition of $P, P P$, and $P S$ wave fronts can be derived from purely kinematic considerations, and pictures of wave fronts so determined can be seen in the book by Cagniard (1962). But figures 2 to 5 display the wave fronts deduced from full dynamical considerations that went into the wave equation and the boundary and initial conditions and the subsequent exact mathematical solution of the problem. While Cagniard could in principle have computed figures comparable to ours, it is doubtful if the necessary numerical calculations were done without digital computers. We have been unable to derive an explicit formula for $K_{P S}$ (on the lines of $K_{P}$ and $K_{P P}$ ) and had to resort to the iterative Newton-Raphson technique to obtain its estimates for each different point using (3).

Rayleigh waves have fascinated seismologists now for almost a century. The work of 


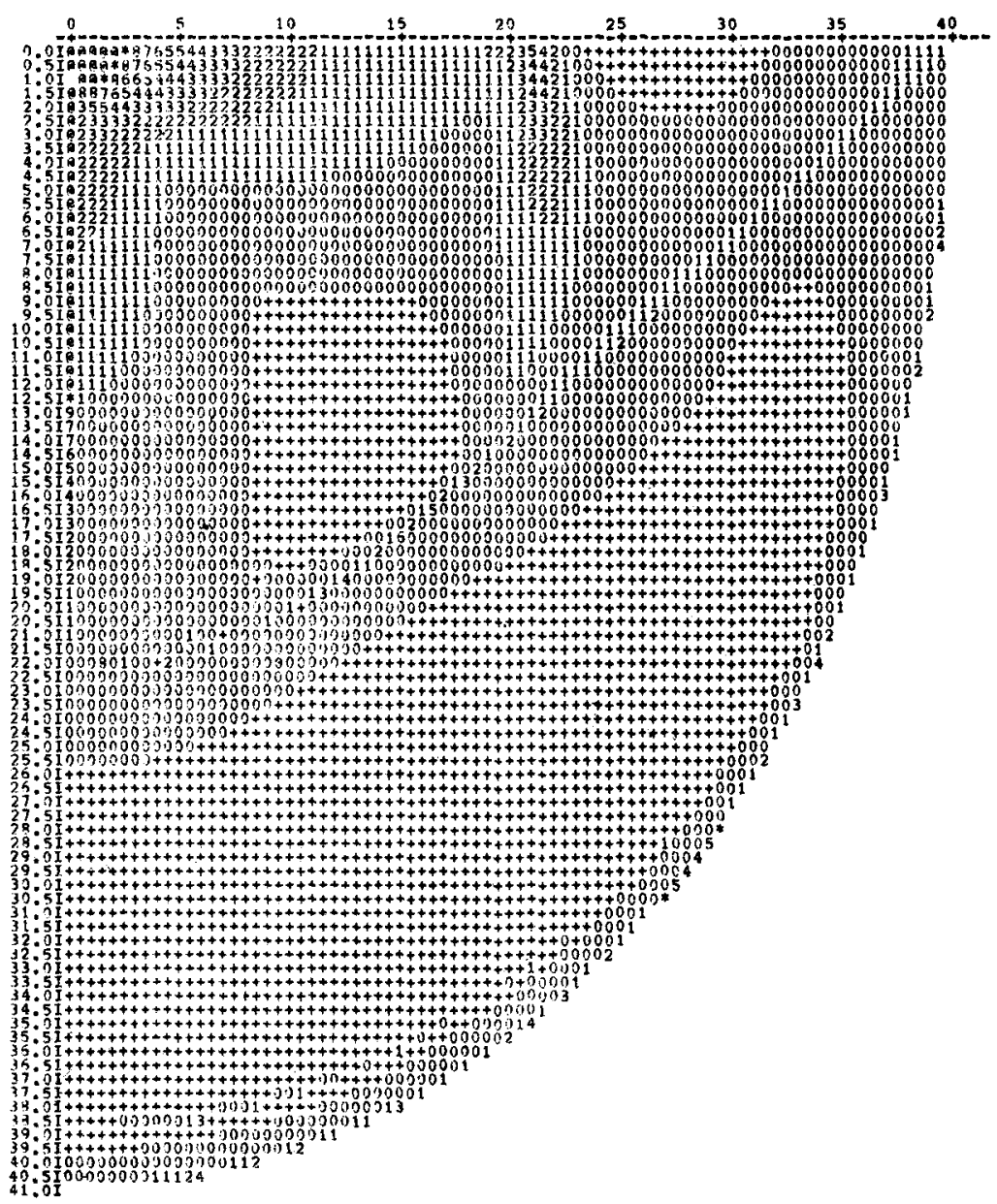

Figure 5. The total displacement $\left(X^{2}+Z^{2}\right)^{1 / 2}$ field.

Lamb (1904) clearly established that a full wave theory of the displacement field due to a transient source in an elastic solid half space contains a contribution with generally the properties of waves investigated by Rayleigh in 1885 (Ewing et al 1957). Over the last 40 years seismologists have turned to the work of Cagniard to find clarifications regarding the behaviour of these waves as revealed in the exact theory. Our results show that the Rayleigh pulse is most pronounced at points near the boundary of the medium where $x$ is approximately $V_{R} t, V_{R}$ being the speed of Rayleigh waves, but its effects are perceived, in however small a degree, throughout the region bounded by the $P P$ wave front but nowhere outside it.

The present results are in accord with the principle of causality, according to which, the medium should not be disturbed until the source has begun to act and emit waves. In other words, since the source in our problem starts to radiate at time $t=0$, the medium should be quiet everywhere until that time. Actually, the exact theory reveals 
that the medium is disturbed only in the region reached by the compressional waves created at the source at time $t=0$.

In short, we have investigated the exact solution of the problem of an explosive transient line source in an elastic solid half space at points within the medium as well as on its bounding surface. We have delineated the region in which the effects of the Rayleigh pulse are to be felt at a given time. Further because of our inability to solve explicitly for $K_{P S}$ we have further narrowed the domain of wave propagation problems for which exact closed form solutions are available, i.e. the domain of problems with exact closed form solutions excludes the present problem when the subsurface points of the medium are considered.

\section{References}

Bhandari S K 1982 Exact solution for the response of a homogeneous, isotropic, perfectly elastic solid half space to a buried explosive line source, M. Tech. dissertation, University of Roorkee

Cagniard L 1962 Reflection and refraction of progressive seismic waves (translated by E A Flinn and C H Dix) (New York: McGraw-Hill)

Ewing M, Jardetzky S and Press F 1957 Elastic waves in layered media (New York: McGraw-Hill)

Garvin W W 1956 Proc. R. Soc. London A234 528

Lamb H 1904 Philos. Trans. R. Soc. London A203 1 